\title{
3D STEM for Imaging Biological Samples and Nanoparticles
}

\author{
A.R. Lupini, ${ }^{*}$ S. J. Pennycook, ${ }^{*}$ and N. de Jonge**
}

* Materials Science and Technology Division, Oak Ridge National Laboratory, Oak Ridge, TN 37831-6031, ** Vanderbilt University School of Medicine, Department of Molecular Physiology and Biophysics, TN, Nashville, 37232-0615

The recent successful correction of spherical aberration in scanning transmission electron microscopy (STEM) [1] allows the use of a larger convergence angle, which causes a decrease in the depth of field (DOF). This change is of interest because the DOF is reduced by about an order of magnitude, meaning that a series of images can be recorded at different focal values, effectively obtaining a series of slices through a sample and allowing three dimensional (3D) images to be obtained [2-4].

This depth-sectioning mode (3D STEM) allows extremely high lateral resolution with a vertical resolution of several nanometers and has displayed sensitivity to single atoms [2-3]. Although detection limits and beam induced motion can limit the precision, single heavy atoms can be located in all three dimensions on materials such as catalysts [2] or semiconductors [3, 4]. However, for large biological samples that might be several tens of microns across, might be susceptible to beam damage, and possess interesting structures that offer only very weak contrast, the imaging requirements may be very different [5]. In this application it is possible to use heavy nanoparticles to label specific proteins [6,7]. These nanoparticles are typically $1-5 \mathrm{~nm}$ in diameter and are selected to have a large atomic number $Z$ such that they will be visible through a relatively thick carbon-based sample. In these samples, an improvement in the (relatively poor) depth resolution of the 3D STEM technique might be desired, even at the expense of degrading the lateral resolution.

For an aberration-free probe increasing the aperture size will reduce the DOF, but in practice the DOF is usually limited by aberrations, analogously to the $2 \mathrm{D}$ resolution. Numerical calculations [8] suggest that the aperture angle that optimizes the 3D DOF will be close to the same value that optimizes the 2D probe size under typical conditions. However, when imaging extended labels, the vertical resolution does not always correspond to the traditional DOF, but is limited by the shadow due to the finite label size. Fig. 1 is calculated for a STEM without an aberrationcorrector and shows the DOF for the probe and the full width at half maximum (FWHM) in the vertical direction for a particle size of $5 \mathrm{~nm}$. It can be seen that although the DOF is optimized at an aperture angle of about $9 \mathrm{mrad}$ (close to the optimal angle for 2D resolution), the vertical FWHM of the $5 \mathrm{~nm}$ particles is over $660 \mathrm{~nm}$. Increasing the aperture size to $17 \mathrm{mrad}$ degrades the DOF and the potential vertical FWHM for single atoms, but improves the vertical FWHM for the $5 \mathrm{~nm}$ particles to about $400 \mathrm{~nm}$. Note that the position of the maximum intensity is displaced vertically.

A focal series might also be used together with a tilt series to combine the advantages of both techniques. This combination has obvious applications in samples where the vertical extent is greater than the DOF, but more advanced reconstructions may require new algorithms to be implemented. [9] 
References

[1] O.L. Krivanek, N. Dellby \& A.R. Lupini, A.R., Ultramicroscopy 78(1999) 1.

[2] AY. Borisevich, A.R. Lupini \& S.J. Pennycook (2006). Proc. Natl. Acad. Sci. 103(9), 3044.

[3] K. van Benthem et al., Appl. Phys. Lett. 87 (2005) 034104-1.

[4] A.R. Lupini et al Microsc. Microanal. 15 (009) 441.

[5] N. de Jonge et al., 3-dimensional aberration corrected scanning transmission electron microscopy for biology. in Nanotechnology in Biology and Medicine, ed. Vo-Dinh, T., pp. 13.113.27 (CRC Press, Boca Raton, 2007).

[6] N. de Jonge et al., Microsc Microanal 16 (2010) 54.

[7] Y. Xiao et al., Science 299 (2003) 1877.

[8] A.R. Lupini \& N. de Jonge, Microsc. Microanal. submitted (2011).

[9] Research sponsored by the Division of Materials Sciences and Engineering, Office of Basic Energy Sciences, U.S. Department of Energy, at Oak Ridge National Laboratory (SJP) and by NIH grant R01GM081801 (ARL \& Nd).

(a)

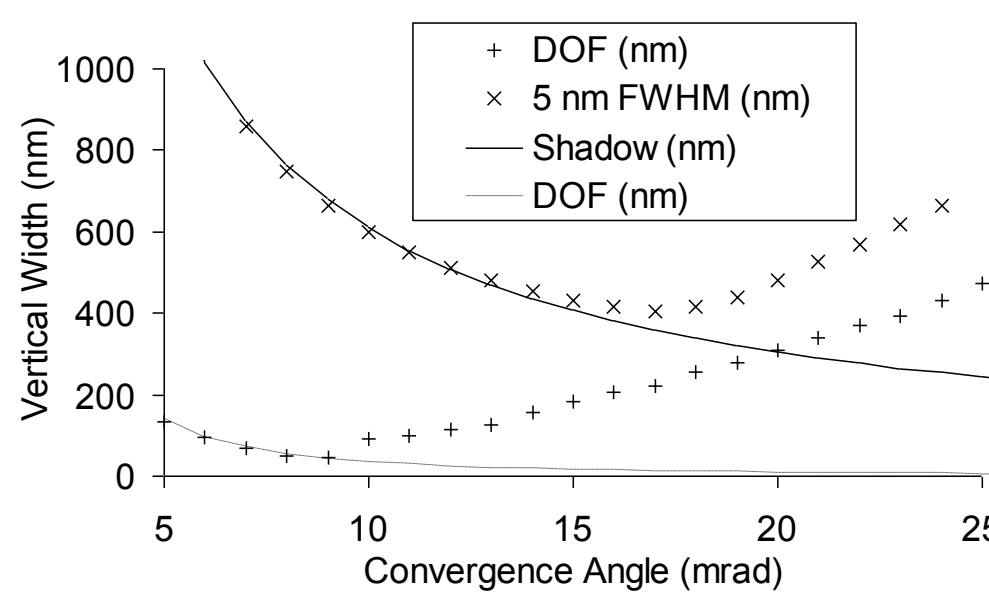

(b)

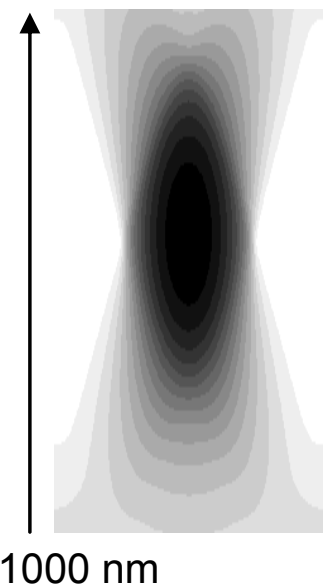

(c)

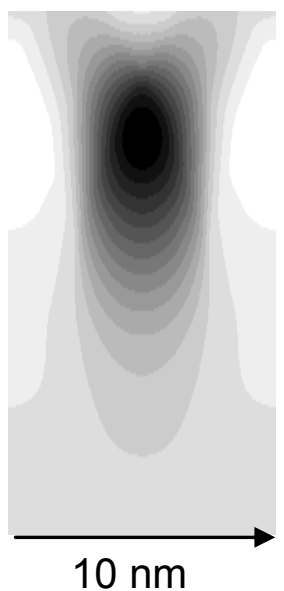

FIG. 1. (a) The vertical DOF for the probe calculated as in [8], and the vertical FWHM for $5 \mathrm{~nm}$ particles calculated by convolution. Analytical estimates for the DOF and the shadowing effect are shown as lines. (b) Cross-section through the convolution of a $5 \mathrm{~nm}$ diameter nanoparticle with probe generated with a 9 mrad convergence angle. (c) Same with a $17 \mathrm{mrad}$ angle. Parameters used are for an uncorrected microscope: $C_{3}=1.3 \mathrm{~mm}, C_{5}=0.15 \mathrm{~m}, 300 \mathrm{kV}$. 